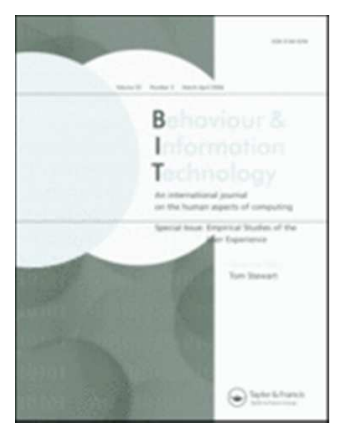

\title{
A Framework for Informing Consumers on the Ecological Impact of Products at Point of Sale
}

\begin{tabular}{|r|l|}
\hline Journal: & Behaviour \& Information Technology \\
\hline Manuscript ID & TBIT-2016-0413.R1 \\
\hline Manuscript Type: & Original Research Study - Full Article \\
\hline Keywords: & $\begin{array}{l}\text { ecological impact, consumer decision, augmented reality, recommender } \\
\text { system }\end{array}$ \\
\hline \multicolumn{2}{|l}{} \\
\hline
\end{tabular}

SCHOLARONE ${ }^{m}$

Manuscripts 
To appear in Behaviour \& Information Technology

Vol. 00, No. 00, February 2018, 1-28

\title{
A Framework for Informing Consumers on the Ecological Impact of Products at Point of Sale
}

(Received September 2016; revised February 2018)

\begin{abstract}
The use of intelligent information technologies has the means to provide ecological information just-intime, thus alleviating consumers' cognitive burden at the time of purchase. We propose a computational framework for supporting consumer awareness of the ecological impact of products they consider purchasing at point of sale. The proposed framework permits consulting multiple information sources through diverse access interfaces, combined with a recommendation engine to score product greenness. We evaluate our approach in terms of usability, performance, and user-influence tests through two conceptual prototypes: an online store and an augmented reality interface to use at physical stores. Our findings suggest that providing ecological information at the time of purchase is able to direct consumers' preference towards products that are ecological and away from products that are not; consumers also express willingness to pay slightly more for ecological products. The experimental results obtained with the interface prototypes are statistically significant.
\end{abstract}

Keywords: ecological impact; consumer decision; augmented reality; recommender systems

\section{Introduction}

There is a global need for higher awareness of the importance of nature and climate preservation (Coelho, de Castro, and Alcides Gobbo Jr. 2011). Even though this is a complex task with several stakeholders, simple consumer choices in everyday life can contribute (purchasing recyclable products, using renewable energy sources, etc.). However, cultural views and scientific literacy (i.e., the ability to interpret scientific results) affect the interpretation of information on ecological issues (Kahan et al. 2012). Selecting green products may seem simple at a first glance, but consumers in general are not necessarily aware the ecological impact of products they choose at the time of purchase; particularly novice consumers struggle identifying eco-labels (Thøgersen, Haugaard, and Olesen 2010). 
Gan et al. (2008) confirm in their study that ecologically conscious consumers are more likely to make ecologically sound purchase decisions. Accurate knowledge about product ecological compatibility is nevertheless necessary for ecologically-conscious consumers to make sound decisions at the time of purchase (Roberts and Bacon 1997). The cognitive burden at the time of purchase (that is, the strain to recall pertinent aspects and carrying out an evaluation of available products) is high, especially when there is a considerable number of options to take into account (Miller 1956). It is easier to simply buy "the same as usually" or "whatever is on sale" than making green choices as a consumer. Providing abundant, detailed information at time of purchase actually hinders decision making instead of easing the task (Chen, Shang, and Kao 2009). Customers are also usually rather stressed and time-pressed, which further limits their cognitive efforts (Fram and Axelrod 1990; Aylott and Mitchell 1998).

Some European countries report success stories regarding recycling and eco-labels for naturefriendly products (Galarraga Gallastegui 2002). However, consumers have been found unlikely to transform their behaviour in response to information they receive (regarding energy consumption), explaining their lack of action as having forgotten or it being tiresome and highlights the importance of social context and explicitness in consumer information, as general-level recommendations are ignored in practise by the consumers, even when understood and agreed with in principle (Bartiaux 2008). The same applies in nutritional choices (Moorman 1990): upon purchase, the stimulus available at the time outweighs previous nutritional information (only highly motivated consumers would recall nutritional information and take in into account upon deciding a purchase), and consumers with low level of education are particularly prone to ignore any information previously provided.

Information technologies can provide tools to overcome these obstacles; while being pervasive, they can provide relevant knowledge at the moment it is needed, taking into account the user context (Kalnikaitè and Whittaker 2008, 2011). In this work, we propose a framework for generating just-in-time visualisations of relevant ecological information for the products under the consumer's consideration at the time of purchase. The information is displayed through user interfaces for different shopping scenarios (we implemented as conceptual prototypes a browser plugin for e-commerce and an augmented-reality interface for in-store purchases) and integrates various information sources for calculating a score related to the product's greenness; this score, as well as an explanation for it, can be visualised through the user interface. The information sources for the 
framework range from official sites to social networks such as Twitter; consumers show greater interest towards information obtained through internet discussion boards than towards that provided by the manufacturer through marketing (Bickart and Schindler 2001). The main contributions of this work are:

- The design of a computational framework that assesses product greenness by invoking a series of scoring modules, such as general opinion in social networks, identified eco-labels (i.e., national or international certifications regarding the ecological impact of the product, if applicable), identified ecological boycotts, and manufacturing distance; information is received and transmitted through different user interfaces, such as a Web browser and augmented-reality glasses.

- Two conceptual prototypes that implement the user interfaces mentioned above.

- Evidence to demonstrate that the implemented prototypes influence shopper decisions towards greener choices.

The rest of this paper is organised as follows: Section 2 introduces necessary background on recommendation systems and computer vision; Section 3 reviews related work, after which Section 4 describes the framework for product automatic green assessment. Section 5 presents the conceptual prototypes used to validate our approach. Finally, Section 6 discusses conclusions and future work.

\section{Background}

In this section, we review some of the background information necessary for the remainder of this work, specifically terminology and conceptual definitions.

\subsection{Eco-friendly consumer behaviour}

Ecologically responsible consumers prefer organic products, avoid aerosols, choose locally produced goods, and bring reusable bags to the supermarket; the degree of fulfilment with these activities classifies consumers into four categories: committed environmentalists, mainstream environmentalists, occasional environmentalists, and non-environmentalists (Gilg, Barr, and Ford 2005). Factors that may drive a consumer towards the purchase of environmentally friendly products are numerous and not fully understood, although published literature on consumer behaviour as such is abundant — a survey of literature regarding numerous empirically observed factors is given by 
Joski and Rahman (2015).

The following four factors are empirically found to have an influence in green marketing: eco labels that seek to ease understanding of ecological information, including the capability of building an emotional relationship with customers, product value and attractiveness, and green advertisement components (Azad et al. 2013).

Consumers are reported to struggle to understand eco labels and to assess the credibility of ecological information on product packaging; only those consumers who express the high interest in ecology are unhindered (Carrero Bosch and Valor 2012). An analysis of factors affecting green consumption in Australia found that consumers are generally sceptic about companies risking their profitability in exchange for a greener production and that aspects such as labels, packaging, and ingredients do not affect consumer perception on green products. (D'Souza et al. 2006) In that study, consumers reported to experience difficulty understanding eco labels and the only factor found to make a positive impact on green product perception was consumers' past experience with the product. A European study reports that only a small fraction of consumers prefer to purchase eco-labelled products (TNS Political \& Social 2013); similarly, consumer awareness appears to diminish at product level and that eco-labels do not play an important part in consumer food choices (Grunert, Hieke, and Wills 2014).

A positive correlation has been reported between environmental opinions and green purchasing behaviour, as well as perceived quality of green products and green purchasing behaviour (Hossein Khorshidi, Gholizadeh, and Naghash 2013), along with a positive correlation between effective green marketing strategies and customers' purchasing patterns for green products (Juwaheer and Pudaruth 2001). Curiously, positive feedback that acknowledges green behaviour is reported to have a negative impact on green choices in the future, whereas consumers who receive negative feedback are more prone to become green (Longoni, Gollwitzer, and Oettingen 2014). However, positive cues for common ecological behaviours (e.g. avoidance of littering) results in a more frequent selection of eco-friendly products (Cornelissen et al. 2008).

The relationship between price and perceived quality in consumer decision making appears to be reactionary instead of derived from experience (Tellis and Gaeth 1990); this finding highlights the importance of presenting any relevant information at the time of purchase instead of expecting the consumer to remember and learn. Similarly, a study of the effect of information regarding climate change in the decision making of tourists found consumers improperly informed of such 
ecological concerns (Góssling et al. 2012). Also consumer lifestyle has been reported to affect purchase decisions in Taiwan: a tailored marketing strategy is needed for each lifestyle to promote green consumption (Wang 2012b,a).

Several recent studies have focused on consumer behaviour in developing countries, specially those with a high population (e.g. India). A positive relationship between social perception (green self-identity, for instance) and eco-friendly consumer behaviour has been reported (Barbarossa and De Pelsmacker 2016); other positive factors include values such as biospheric values (Nguyen, Lobo, and Greenland 2016, 2017), a positive attitude, and moral. The more committed consumers, including youngsters (Prakash and Pathak 2017), are even willing to pay more for eco-friendly products and accept their shortcomings. In general, a greater eco-friendliness is observed when there is a greater knowledge about environmental issues (Biswas and Roy 2015; Yadav and Pathak 2016). However, it has also been reported that, in some contexts, eco-friendly consumers have different motives (other than care for the environment) for their choices; this has been the case for eco-city dwelling in Singapore (Flynn et al. 2016). Other recent studies have surveyed the types of most popular green products, revealing that preference is given to those with a relative comparative advantage (Fraccascia, Giannoccaro, and Albino 2018).

\subsection{Recommender systems}

The purpose of a recommender system is to detect items of interest for a target user. Recommendations can be non-personalised or personalised: while the former are centred on suggesting "hot" items (e.g. top-ten songs), the latter collect particular user preferences (either implicitly or explicitly) and recommend items based on these preferences. The two classical approaches for making recommendations are content-based systems and collaborative filtering. Content-based systems take into account items that were given high ratings by the user and search for other items similar to these, whereas collaborative filtering searches for items that were given high ratings by users that are similar to the target user. Other approaches include demographic, knowledge-based, socialbased, context-aware, and hybrid (Ricci, Rokach, and Shapira 2011; Adomavicius and Tuzhilin 2011; Bobadilla et al. 2013). Recommender systems normally produce a sorted list of items, where order determines the items' likelihood to be highly rated by the target user.

Currently, recommender systems have focused on user profiling (Al-Shamri 2016), as well as social and trust-centric recommenders, which take advantage of friendship networks to provide 
recommendations with a higher level of reliability or trust (Moradi and Ahmadian 2015). Furthermore, advances in machine learning (deep learning, for example) have improved the state-of-the-art by approaching the cold-start problem (Elahi, Ricci, and Rubens 2016) and increasing product acquisitions (Cheng et al. 2016). Moreover, it has been stated that recommender agents influence consumer behaviour (Huseynov, Huseynov, and Özkan 2016). However, there is still work to do, for instance, in the area of interactive recommender systems (He, Parra, and Verbert 2016) — i.e., systems that combine recommendations with visualisation. Another interesting direction, which can also impact product recommendations, is the incorporation of open linked data (Musto et al. 2016).

\subsection{Image processing}

Computer-vision techniques enable extracting information from product packaging with a digital camera. There are several different approaches that can be used individually or complimentary to one another for processing images of product packaging. Optical character recognition (OCR) identifies text in the input images and attempts to recreate a digital textual version of the fragments of text found in an image (Jung, Kim, and Jain 2004). The extracted text can then be processed to identify information regarding the ingredients, the manufacturer, the geographical location in which the product was manufactured, and so forth. Feature detection seeks to match graphical entities present in an image to a pre-processed database of images (logotypes of manufacturers or brands, eco-labels, etc.) (Tuytelaars and Mikolajczyk 2008). Thirdly, decoding bar codes and their two-dimensional variants QR codes (Ohbuchi, Hanaizumi, and Hock 2004) recovers the information embedded in the code - in the case of bar-codes, this is usually a series of digits (which then needs to be consulted in a product database, some of which are available online), whereas for the QR codes the information contained is commonly a URL pointing to a website related to the product, but could also contain any other textual or numeric data.

The potential sources of the images to process are diverse: in the case of e-commerce, there are often product photographs on the seller's website, whereas a person shopping in a store can use a smart-phone with a camera to "scan" products of interest and then visualise the obtained information on the screen of the smart-phone (von Reischach et al. 2009). Commercial wearable augmented reality (AR) headsets are increasingly available and make it possible not only to capture in images the objects that a person is presently viewing but also allows for a graphical overlay of 


\section{Related work}

Our present work aims at making ecological information more readily available and easier to interpret for consumers that are not motivated enough to inform themselves beforehand. Our main goals are providing access to information on a level of detail that the consumer may easily control at the time of purchase to lower the cognitive burden of remembering and understanding the factors involved in determining whether purchasing a specific product is an ecologically friendly choice and providing visual feedback on the eco-friendliness of the choices made at the time of purchase. Our proposal is effectively a shopping assistant that recommends eco-friendly products, with an optional augmented-reality interface for in-situ purchases.

The use of AR in ecological content is scarce at present. Kamarainen et al. (2013) use of AR for ecological awareness of students during field trips, whereas Tarng and Ou (2012) apply AR for teaching ecology to students. Lu and Smith (2007) use AR to visualise three-dimensional models of products, for example to determine which couch would look good in the user's living room. We have not encountered any literature applying AR for informing consumers at time of purchase over ecological aspects of the products. Our proposed system differs from the aforementioned works by recognising images from the product on the user's field of vision and providing information related 
to the ecological impact of this product.

In the remainder of this section, we review related work on shopping assistants on (green) product recommenders.

\subsection{Shopping assistants}

The process of consumer decision making in an interactive online setting is studied by Häubl and Trifts (2000), finding that recommendation systems have a substantial impact in consumer choices. Also Park, Lee, and Han (2007) report that online consumer reviews effect consumer behaviour: a purchase is more likely in the presence of positive reviews. Laros and Steenkamp (2005) document the power of emotions over purchase decisions.

Already in 2002, Menczer, Street, and Vishwakarma (2002) proposed a web-based agent that can learn shopping preferences, search for items that match them, and guarantee user privacy. More recently, Bhattacharya et al. (2012) implemented a mobile shopping assistant that can be accessed using a Web or mobile-phone interface features that were determined with shopper questionnaires and include a natural language processing module for extracting products from shopping lists, predictive text input for making such lists, an engine for retrieving products given their name or description, a product and promotion recommender, and an indoor positioning to help locate products in the store - the system's inception in a supermarket helped increase sales.

Kourouthanassis, Giaglis, and Vrechopoulos (2007) discuss the potential of ubiquitous systems in affecting the shopping experience of users and conclude that it is possible to positively influence emotions at time of purchase by creating an entertaining experience. Their work differs from the one proposed in this paper as their chosen technology is a tablet device and the products are recognised by RFID instead of computer vision and product recognition. Also, their goal differs as they seek to help the user to be more economical, whereas our aim is influencing the ecological behaviour of the user.

There are also several patents related to shopping assistants. The patent by Burke, Lalwani, and Thong (2003) provides product location and reviews by using a mobile device with a bar-code reader connected to a database for product identification. Another patent by Kern and Abbass (2008) describes a system that emits purchasing recommendations based on a correlation between user profiles and products. The patent of Bravo (2014) introduces a shopping assistant based on AR; this assistant guides its user within the store to the location of a specified product. Likewise, 
the patent by Martin (2012) permits to search for products, either on a mobile or augmentedreality device, and receive the locations (stores) that have these products in inventory. Neither of these patents attend aspects of product recognition via computer vision (as we do) nor ecological concerns.

The shopping assistants reported in the literature differ from our proposed framework mainly in terms of the ecological orientation of the framework and the use of scoring modules to produce a recommendation. Furthermore, our system - at this point — is not designed to automatically infer user preferences from the queries and the interactions, although future work includes registering user actions with the intent of producing recommendations.

\section{2. $\quad$ Product recommenders}

Existing product-recommendation systems includePromotionRank (Nurmi et al. 2014) that ranks and recommends grocery product promotions according to a personal shopping list. The process initiates with the creation of a candidate product pool, which is then expanded with related products, and then category rank scores are calculated for the pool, after which the) promotion ranking takes place. With focus on environmental recommendations, Lee, Huang, and Hwang (2009) propose a system that gradually suggests products with a higher greenness, based on eco-labels and sentiment words, but - in contrast to our framework - it only considers an online shopping interface. Fang and Sun (2016) propose and evaluate three user interfaces to stimulate household water saving, focusing on emotional response.

Liangxing and Aihua (2010) provide a hybrid recommender system for apparel retailing customers that uses RFID technology to detect member cards and generate recommendations based on products that are similar to past purchases and products that other clients have bought. Aciar et al. (2007) score the quality of a product in terms of consumer reviews, using text-mining techniques to extract reviews that are then mapped into an ontology with rule-based classification techniques. A ranking, considering the reviewer's level of expertise, is computed for each product.

Recommendation systems reported in the literature rarely apply computer-vision techniques; furthermore, our proposed work is aimed at multiple interfaces and permits combining multiple scoring modules to produce the recommendation. 
Figure 1. Principal components of the proposed ecological product-recommendation framework.

\section{Proposed framework}

With the intent of helping consumers to make greener choices at the time of purchase (while at the same time alleviating the burden of keeping in mind every aspect related to a product's greenness), we propose the design of a computational framework that produces both global and specific scores for the greenness of a given product. Our proposed framework, consequently, takes a product as input (e.g. as an image or unique identifier) from a user interface, breaks down the input information into a set of usable keywords (which we shall refer to as query), evaluates the product's greenness using different scoring modules that take the query as input, and returns the score (as well as an explanation that consists of an individual score per module) as a smart visualisation by means of the same user interface. Figure 1 visualises this structure, and the principal operational entities are discussed in this section.

As in all human-computer interaction, the possibility of incorrect input due to distractions or misunderstandings is omnipresent. Hence the output needs to clearly identify how the input was interpreted (for example, if a query was assumed to refer to a specific product, an image of the product can be included in the response so as to identify the product that the results refer to) and, ideally, a confidence score (on a numeric scale, an icon-based scale or a background colour, for example) on how precise and exhaustive the results are also needs to be presented to the user, together with an option to refine the query in case the result was not the desired one. When multiple potential matches are found to a user's query, the list of the possible results needs to be ordered by relevance (similar to a search engine's results) for the user to select from.

\subsection{User interfaces}

Providing in-situ information can be efficient if delivered right (Bird, Kalnikaité, and Rogers 2011), although existing works have mainly been limited to overall recommendations. Ecological considerations, which are the main focus of the present work, often rely on multiple factors (Gan et al. 2008), many of which require a more elaborate presentation. As reported by Pancer, McShane, and Noseworthy (2017), isolated visual cues do not work well, while Lee, Bhatt, and Suri (2018) find that limiting to a small subset of aspects results in a negative consumer attitude. Relevant factors include but are not limited to the following: 
Origin: Can the product be considered locally produced? How far away the product was produced and how has it been transported to the point of sale; also, for online retail, from where it will be delivered to the customer and by which means of transport?

Raw materials: What are the ingredients the product is made of or grown with? What is the packaging made of? (The effect on how green the packaging appears to be on how green the product is perceived to be has been studied by Magnier, Schoormans, and Mugge (2016) for food products.) How sustainable are these raw materials? From where are the raw materials obtained and what are the ecological impacts of their recollection? If the raw materials are renewable, how rapid is this renewal?

Manufacturing: How sustainable is the process used to manufacture the product (or cultivate the produce)? What energy sources are used? What type of waste is created by the process? Does the process contaminate the environment at the site of production?

Useful life and reuse: How long will the product be useful? Will some parts of the product or its packaging be reusable after the useful life of the product itself ends?

Recycling: Will the product or its packaging result in waste? How much and what type of waste? How much of it can be recycled, where, and how? If and how recycling is to be carried out at the place of residence of the consumer: is there a drop-off spot, a collection service, is the waste biodegradable, etc.

Certifications: Which organisations, if any, have verified the eco-friendliness of some of these aspects of the product — that is, what eco-labels does the manufacturer, the ingredients, the product and/or the packaging have?

Also, as many modern products are in fact assembled from multiple components that have been manufactured in distinct locations with different processes, the importance of each component in the perceived greenness of a product varies (Gershoff and Frels 2015). In general, it is hard to communicate ecological impact to people with little expertise in the field (Brunson and Reiter 1996).

Regarding the input of the data to identify a product of interest through the interface, we consider as the main options photographs of packaging, bar codes or a set of typed key words. In each case, a list of suggested matches needs to be presented for the user to choose from, similarly to a modern web search engine. As the primary focus of the proposed framework is to support the user 
at the time of purchase, in an in-store context this often means that the product is physically at hand or on a shelf in front of the user - items such as fresh produce in a store will not necessarily be packaged with identifying marks, but most countries require stores to display a sign with details of the origin for items such as fruits and vegetables and a photograph of such a sign can also serve as input. For online use, we assume that a user attempting to make a purchase decision is presently looking at a web page with the product information and can provide information such as a screen capture, a copy-paste of some text, or even the URL of the page as input to specify the product in question (the latter requiring some parsing to identify the product information). We are aware that some types of products and services will fall outside the scope of a framework of this type, but we expect it to be feasible to correctly identify a grand majority of supermarket items and household products available through web stores.

The proposed framework allows for an unlimited number of user-interface modules that permit the consumer to input products and access the generated ecological recommendations. We have implemented two user-interface prototypes:

\section{Augmented-reality interface (Espinosa Ceniceros, Schaeffer, and Garza Villarreal} 2014). Either through a mobile phone or an AR-headset, the interface accesses the camera to identify the product at the centre of attention of the user and then overlays visual representations of the obtained ecological recommendations.

Web-browser plugin (Urbina Coronado, Schaeffer, and Garza Villarreal 2014). An application developed for the Chrome web browser that parses the pages that a consumer is viewing in a web store and, upon mouse-over on a product, adds a visualisation of the ecological recommendation of the identified product.

\section{2. $\quad$ Processing modules}

User interfaces represent the product to evaluate either with images (in the case of the AR-headset or the mobile phone) or a textual description (in the case of the Web plugin). This requires the addition of processing modules to improve the speed and accuracy of product recognition and classification. In general, the framework includes (but is not limited to) several image processing modules, a natural language processing (NLP) module, and — optionally - a geo-localisation module. Let us describe each one: 
A module for Natural Language Processing (NLP) is necessary to determine the meaning of any retrieved text, required for its processing (particularly., whether the information contained is neutral, negative, or positive in terms of ecological impact). Sources of text include the OCR module, the website of the producer and/or the product as well as information on product review websites, and the opinions expressed by users in social networks.

A geo-localisation module, upon extracting from the user interface the location of the user, compares it to the location of manufacture (extracted from the packaging or by querying product information repositories) and determines whether a specific production location is local or not to that particular user at that specific moment.

An image processing module is necessary to detect the product at hand and its main components. As such, image processing modules have the capacity of recognising the image of a product (what product it corresponds to and what are the words contained in the product's label), an eco-label, a QR code, etc. These modules receive images from the user interface and retrieve information regarding the products that appear in the photographs taken with the phone or the AR glasses. Examples of these modules include the following:

A logo-recogniser module aids on the identification of a product (brand, producer) and its ecocertifications. The module compares elements present in the input image to a database of known logotypes using feature detection. This permits the incorporation of the information of the presence and absence of such symbols in the computation of an ecological score, no longer requiring the user to recognise and interpret their meaning.

A code scanner module retrieves product information which otherwise remains cryptic to the consumer. The module interprets bar codes and two-dimensional QR codes present in the image. The product identification numbers extracted in this manner can be used to consult in-store and online databases for product information, which can also be incorporated into the computation of ecological scores.

Finally, the Optical Character Recognition (OCR) discovers other relevant product features: it detects any textual information present in an image and converts it into text in order to extract detailed product information. 


\subsection{Query handler}

A query handler is a module that takes care of the communication between the processing modules (image, NLP, geo-localisation) and the green recommendation engine. A query aggregator receives information retrieved by the processing modules and combines it into a bag-of-words representation that acts as a product query, containing labelled subsets of data such as names of ingredients, place of production, brand name, bar code, company name, and names of ecological certifications present in the packaging. Each subset is a separate bag-of-words that is accompanied with a label. Possible labels include the ingredients used to manufacture the product, the locations at which the manufacture takes place, and ecological labels awarded to that product or manufacturer, as well as possible active boycotts against the manufacturer.

\subsection{Scoring and recommendations}

Each scoring module is an independent system that provides a numerical score: it receives a set of bag-of-words queries from the recommendation engine, processes that information, and produces a score for each category that produced a match within the information handled by that scoring module, if any (a null response is sent if there are no matches). At the end of this section, we briefly describe the social networking module for the sake of clarity.

An individual scoring module does not need to respond on all categories for all products; it may be specific for a certain type of product or only operate on a limited subset of categories. For instance, there could be a module that applies only for food and drinks.

The information arriving from the query handler is sent through a recommendation engine that acts as a proxy between the query aggregator, the scoring modules, and the user interfaces. The recommendation engine maintains a registry of active scoring modules, sends to each those bags-ofwords that the module has registered to receive (all if no filter is in place), and waits for a determined timeout for each scoring module for a response. The response consists in (category, score) pairs; all arriving pairs are then combined within the recommendation using a scoring function that produces a single score per each category that appeared in at least one scoring module response (options include statistical measures like average or median or fuzzy computation to combine the individual scores, with the option of expressing user preferences in terms of weights). The aggregated (category, score)-pairs are then sent to the user interface that originated the recommendation request.

Figure 2 illustrates the communication between the recommendation engine and the scoring 
Figure 2. The phases of interaction between the recommendation engine and a scoring module of the architecture presented in Figure 1.

modules: initially, a scoring module sends a message to the recommendation engine, indicating its interest to be included as a scoring module in the framework. Then, upon adding the scoring module into the register, the recommendation engine sends back a data model that informs the scoring module of the categories currently employed by the framework and the scoring scale to be used in each category as well as the types of bags-of-words that are included in a query. A scoring module may then inform the recommendation engine which subset of the bag-of-words it will process and to which categories it may provide scores; this is to optimise the performance and avoid sending unnecessary information. Every time the recommendation engine modifies the available query data or the categories, it re-sends a package like this to any registered scoring module.

Upon receiving a query from the query aggregator, the recommendation engine sends it to any scoring module in its registry. If no reply has been received from a scoring module within a predetermined time limit (for example due to network malfunction or discontinuation of the scoring module by its provider), the aggregator increments the inactivity count of that particular scoring module. When a scoring module has an inactivity count above a threshold, it is removed from the registry (until it re-registers); the inactivity count is reset to zero as soon as a scoring module replies to a query. The query replies are then combined by the recommendation engine as input to the scoring function.

To speed up the retrieval, a cache module is recommended to store information on recently queried products in case the user wishes to reconsider among options, thus avoiding the computational overhead of having to consult the same information again.

Each reply that a scoring module produces includes an identifier (a unique ID within the responses of that module in a 24-hour time period) that can be used if the user interface that generated the query requires access to a textual description of what information contributed to the score. If the user selects to consult the details of a specific score, this ID can then be used by the recommendation engine to retrieve details without a need for the scoring module to recompute this information. The details are not sent automatically with the original response to minimise communication overlay, as the number of modules and categories may increase and the amount of detail in these responses may grow significantly; this would be a problem for mobile-data access. The detailed information is simply a HTML5 document that the user interface will then display 
in a web-view beside the score-visualisation gadget upon user request. We recommend combining colours and symbols for visualising to the user the scores - merely displaying a number is slower to grasp for a user operating in a context of constant distractions such as supermarket, and making the user read a text on each product would risk interrupting the flow of decision-making.

Example: Social networking module. To illustrate the function of the scoring modules, let us describe the design of the social networking scoring module, which is one of the modules we propose. In general, this module would gather opinions from the product's greenness, rate each opinion, and provide a general score that reflects the overall opinion with regard to the eco-friendliness of the product. Its main task consists of retrieving comments (e.g. tweets) about the product, which can be easily performed via automated tools, such as the Twitter API ${ }^{1}$. These tools permit to download a considerable number of real-time comments given a set of keywords (in this case brand, product, and eco-related keywords).

A second important task is to distinguish between opinions and objective text, while also being able to distinguish between positive and negative opinions. Currently, this is possible with methods from sentiment analysis (Liu 2012). The simplest approach is to use a dictionary that contains polar words, i.e., words that express a positive or negative opinion; this dictionary is called lexicon. Normally, in a lexicon each polar word has a weight that indicates if it is negative (weight less than zero) or positive (weight greater than zero). If a word is not found in the lexicon, its weight is zero, which means it is neutral. The rating of each comment is the sum of its weights. The final step consists of calculating an overall score that will be returned by the module. In part, this can be done by combining the individual ratings of the retrieved comments.

Another issue consists of how to ensure content quality and correctness. Reputation systems usually handle environments where the existence of controversial content is possible; it is not uncommon to see this kind of system applied to social media. The traditional approach for reputation systems consists of voting or rating contents to establish their quality or confidence. In Wikipedia, for example, content-based reputation systems based on contribution persistence have been proposed (Adler and de Alfaro 2007); consequently, contributors whose content frequently gets edited have a low confidence score. Reputation can also be established via metadata collection, virtual trophies - these have also been used in eco-friendly applications (Massung et al. 2013) —, feedback, profiles, bot identification, statistical filtering, user promotions, and runtime analytics (Daniel et al.

${ }^{1}$ Available at https://dev.twitter.com/overview/api. 
2018). More sophisticated methods include logical argumentation (Sklar et al. 2016), which is an extension of automated reasoning and negotiation in an agent-based environment to reach agreements when conflicting information is presented. However, it has been stated that crowd-sourcing itself is a truth-converging tool (Goodchild and Li 2012).

\section{Experiments and Results}

In this section we describe experiments carried out with two prototypes - one for web stores and another for regular retail using augmented reality. Both evaluations indicate that providing a consumer with information regarding the ecological impact of products affects their behaviour.

\subsection{Methodology}

We concentrated on these experiments on groceries, while the proposed platform can also include other types of goods and services. The user groups for our experiments consisted in residents of Northern Mexico, of ages 18 to 40, who had purchased something within the last month and expressed interested in testing a shopping prototype.

The online-store interface was tested by 24 users (12 being a control group that used an regular web store and the other half accessing the web store with our prototype plugin that displays ecological information). For the AR-prototype experiments, twelve people (half of them female) were asked to indicate their brand preferences before and after using the AR prototype that displays ecological information. The recommendation engine for these prototypes was a simple database with simplified, artificial ecological information; the products, however, were real, and hence the users may have previous knowledge and/or opinions regarding these products. The goal was to examine how the prototypes affect the users perceptions of the products.

\subsection{Web store}

With the aim of discovering if just-in-time information orients the consumer towards "greener" choices, in the first experiment, we employed a browser plugin for online shopping. This plugin displays information related to the eco-friendliness of a product (Urbina Coronado, Schaeffer, and Garza Villarreal 2014) by following these steps: 
(1) Once the product is selected, its associated information is identified by the plugin and a query is generated.

(2) A recommendation engine, which runs on a separate web server, receives the query.

(3) The query is processed by the server; if there is ecological information available to fulfil this query, a response is produced with such information.

(4) The plugin places the response within the browser by adapting the styles and sizes on the website.

(5) The user is able to interact with the generated visualisation to access additional details.

In the web-store test, each user was given a list of nine products to buy at the supermarket (Comité fronterizo de obreros 2013). The website to used to perform the test was that of Soriana, a large chain of supermarkets in Mexico, http://www.soriana.com. For each requested product, there were different brands and the choice was up to the user. We registered the prices and the ecological score of the selected products in order to determine whether the users of the plugin were willing to pay more in exchange for greener products; our basic assumption is that such willingness would reflect an increase in their environmental awareness at the time of purchase the willingness to pay a higher price has been demonstrated for organic products (Napolitano et al. 2010) as well as products derived from animals with higher levels of welfare (Napolitano et al. 2008). However, Schnettler et al. (2009) found no apparent willingness to pay more for animal welfare in a developing country, although the origin of a product does outweigh the price.

In our experimenters, users of the plugin systematically chose alternatives with higher ecological scores, even though this increased the price of the purchase, than the control group. The ecological scores with the plugin were on average 41 percent higher, whereas the average increase in price was 8 percent. The difference in the total price of the shopping cart is statistically significant, with a $p$-value of 0.00096 in a pairwise $t$-test performed with $\mathrm{R}^{2}$. It is important to note that there was no actual exchange of money involved and that expressions of willingness to spend more money do not necessarily guarantee that more money would in fact be spent; further experimentation in collaboration with a store is required to examine consumer behaviour (Chandon, Morwitz, and Reinartz 2005).

\footnotetext{
${ }^{2}$ An open-source tool for statistics, available at https://www.r-project.org/.
} 
Figure 3. The difference in the frequency of selection of brands into a set of ten favourites, before and after using the AR prototype (the frequency of selections after the usage minus the frequency of selections of the brand before the usage - positive values indicate a change in favour of the brand whereas negative ones indicate a change against it) versus the ecological score assigned to each brand.

\subsection{Augmented-reality interface for retail}

Our AR prototype detects logotypes when the user holds a product package and displays the ecological score if a match is found in the database (Espinosa Ceniceros, Schaeffer, and Garza Villarreal 2014). The interface hardware was a Vuzix ST AR 1200XLD headset that functions both as output (screen) of the client application and and input (camera) for processing the user's field of vision; it is somewhat heavy and partially limits the field of vision, for which the experience of wearing it (especially for a first time) may divert the user attention towards the headset itself and away from the task.

Each user was requested to indicate ten of their favourite brands from an image containing several brand logotypes, both before and after using the prototype that displays ecological scores. Our goal was to examine whether the preferences are altered by viewing environmental information through the prototype. The results are shown in Figure 3: brands that were displayed with a high eco-scored got selected more after using the prototype (a positive change in selection frequency), whereas low-scored brands got selected less (a negative change in selection frequency). We examined the statistical significance with an analysis-of-variance model in $\mathrm{R}$ and the effect of the score in the change of selection frequency is significant with a $p$-value of 0.0006 .

\section{Conclusions}

We described a computational framework for providing consumers with ecological information on the products they consider buying at the time of purchase. We discuss the structure of such a platform and provide experimental results on two conceptual prototypes, one for a web store and another for in-store purchases that uses augmented reality. We found that timely information of products can affect consumer perception of these and stimulate greener choices; observing such an effect for two distinct user interfaces demonstrates the flexibility of our framework as a tool for ecologically conscious decision making at time of purchase.

According to the results of the experiments, the availability of ecological information at time of purchase can affect the perception of the consumer towards preferring products that are said to 
be ecological, even if this goes against their own previous preference or the cost of the alternative products. Such an increase in the awareness may well result in behavioural changes in consumer behaviour, the measurement of which is left to future work.

The Mexican users in our experiments also expressed a willingness to pay more for ecological products, in agreement with the findings of Prakash and Pathak (2017) in India. Both agree with the studies of Fleith de Medeiros, Duarte Ribeiro, and Noguiera Cortimiglia (2016) with Brazilian consumers and the results of Hussain, Khokhar, and Asad (2014) with Pakistani students; the overall conclusion is that recognised brands and companies that are known to be eco-friendly are preferred. The present study - alongside with the cited studies that were carried out in Brazil, Pakistan, and India - indicates that providing information on the ecological impact in an easyto-absorb fashion is able to steer self-assessed consumer preference towards eco-friendly products even for a higher price.

Future work includes the full-scale implementation of such a system using existing online information sources such as http://world.openfoodfacts.org/. One practical concern is vulnerability to spam and misleading information (the internet has plenty of both). This requires the introduction of administrative mechanisms where a super-user can block abusive scoring modules, as well as a decentralised reputation mechanism where the users can vote for the reliable modules and then the scoring function weighs the scores received from the modules in terms of the reputation of the module, placing a higher importance to the trusted modules.

Personalisation of which scoring modules to take into account and how to weigh between them as well as adjusting the level of detail given by default in the user interface is of importance, as previous studies report high individual differences regarding the amount of information preferred by consumers (Chen, Shang, and Kao 2009). We also believe that incorporating elements of ramification (that is, giving users "points" or "ranks" for their ecological actions, in the way that Facebook encourages users to validate the information provided by businesses and Duolingo rewards users for completing language lessons) could be useful and their effect should be experimentally determined.

Further experiments are needed to determine the relative importance of various ecological factors for the users, differences between visual and textual representation of information for the ecological factors, and possible differences in the response of different user groups (that is, whether factors such as age, gender, education, or socio-economic status affect the way in which the user employs and interprets the ecological information provided by the proposed framework. We also wish to 
measure the degree of knowledge that Mexican consumers have regarding ecological issues and examine whether this is related to their degree of preference toward eco-friendly products.

\section{References}

Aciar, Silvana, Debbie Zhang, Simeon Simoff, and John Debenham. 2007. "Informed recommender: Basing recommendations on consumer product reviews." IEEE Intelligent Systems 22 (3): 39-47.

Adler, B. Thomas, and Luca de Alfaro. 2007. "A Content-driven Reputation System for the Wikipedia." In Proceedings of the 16th International Conference on World Wide Web, Banff, Alberta, Canada, 261-270. New York, NY, USA: ACM.

Adomavicius, Gediminas, and Alexander Tuzhilin. 2011. "Context-aware recommender systems." In Recommender systems handbook, 217-253. Springer.

Al-Shamri, Mohammad Yahya H. 2016. "User profiling approaches for demographic recommender systems." Knowledge-Based Systems 100: 175-187.

Aylott, Russell, and Vincent-Wayne Mitchell. 1998. "An exploratory study of grocery shopping stressors." International Journal of Retail and Distribution Management 26: 362-373.

Azad, Naser, Sina Nobahari, Hamid Bagheri, Mojtaba Esmaeeli, and Mohammad Rikhtegar. 2013. "An exploration study on factors influencing green marketing." Management Science Letters 3 (5): 1369-1374.

Azuma, Ronald, Yohan Baillot, Reinhold Behringer, Steven K. Feiner, Simon Julier, and Blair MacIntyre. 2001. "Recent advances in augmented reality." IEEE Computer Graphics and Applications 21 (6): 34-47.

Barbarossa, Camilla, and Patrick De Pelsmacker. 2016. "Positive and Negative Antecedents of Purchasing Eco-friendly Products: A Comparison Between Green and Non-green Consumers." Journal of Business Ethics 134 (2): 229-247.

Bartiaux, Françoise. 2008. "Does environmental information overcome practice compartmentalisation and change consumers' behaviours?." Journal of Cleaner Production 16 (11): 1170-1180.

Bhattacharya, Sourav, Patrik Floréen, Andreas Forsblom, Samuli Hemminki, Petri Myllymäki, Petteri Nurmi, Teemu Pulkkinen, and Antti Salovaara. 2012. "Ma\$\$iv€ - An Intelligent Mobile Grocery Assistant." In Proceedings of the 8th International Conference on Intelligent Environments, Guanajuato, Mexico, 165-172. IEEE.

Bickart, Barbara, and Robert M. Schindler. 2001. "Internet forums as influential sources of consumer information." Journal of Interactive Marketing 15 (3): 31-40.

Bird, Jon, Vaiva Kalnikaité, and Yvonne Rogers. 2011. "The Augmented Shopping Trolley: An Ambient Display To Provide Shoppers with Non-Obvious Product Information." In Proceedings of the 2nd International Workshop on Persuasion, Influence, Nudge ${ }^{3}$ Coercion Through Mobile Devices, Vancouver, 
Canada, 3-6.

Biswas, Aindrila, and Mousumi Roy. 2015. "Green products: an exploratory study on the consumer behaviour in emerging economies of the East." Journal of Cleaner Production 87: 463-468.

Bobadilla, Jesus, Fernando Ortega, Antonio Hernando, and Abraham Gutiérrez. 2013. "Recommender systems survey." Knowledge-Based Systems 46: 109-132.

Bonetti, Francesca, Gary Warnaby, and Lee Quinn. 2018. "Augmented Reality and Virtual Reality in Physical and Online Retailing: A Review, Synthesis and Research Agenda." In Augmented Reality and Virtual Reality: Empowering Human, Place and Business, edited by Timothy Jung and M. Claudia tom Dieck, chap. Augmented Reality and Virtual Reality, 119-132. Cham, Switzerland: Springer International Publishing.

Bravo, Luis Eduardo. 2014. "Method and apparatus for augmented reality shopping assistant." US Patent 8,626,611, https://www.google.com/patents/US8626611.

Brunson, Mark W., and Douglas K. Reiter. 1996. "Effects of Ecological Information on Judgments about Scenic Impacts of Timber Harvest." Journal of Environmental Management 46 (1): 31-41.

Burke, Raymond Robert, Avanti Lalwani, and John Thong. 2003. "Evaluative shopping assistant system." US Patent 6,604,681, http://www.google.com/patents/US6604681.

Carrero Bosch, Isabel, and Carmen Valor. 2012. "La relación del consumidor con el etiquetado responsable." Responsabilidad Social de la Empresa 4 (1): 79-104.

Chandon, Pierre, Vicki G Morwitz, and Werner J Reinartz. 2005. "Do intentions really predict behavior? Self-generated validity effects in survey research." Journal of Marketing 69 (2): 1-14.

Chen, Yu-Chen, Rong-An Shang, and Chen-Yu Kao. 2009. "The effects of information overload on consumers' subjective state towards buying decision in the internet shopping environment." Electronic Commerce Research and Applications 8 (1): 48-58.

Cheng, Heng-Tze, Levent Koc, Jeremiah Harmsen, Tal Shaked, Tushar Chandra, Hrishi Aradhye, Glen Anderson, et al. 2016. "Wide \& Deep Learning for Recommender Systems." In Proceedings of the 1st Workshop on Deep Learning for Recommender Systems, Boston, MA, USA, 7-10. New York, NY, USA: ACM.

Coelho, Tatiene M., Rosani de Castro, and José Alcides Gobbo Jr. 2011. "PET containers in Brazil: Opportunities and challenges of a logistics model for post-consumer waste recycling." Resources, Conservation and Recycling 55 (3): 291-299.

Comité fronterizo de obreros. 2013. "Lista de productos básicos." [Date of access: 2014-26-06], http: //www. cfomaquiladoras.org/listaproductosbasicos.es.html.

Cornelissen, Gert, Mario Pandelaere, Luk Warlop, and Siegfried Dewitte. 2008. "Positive cueing: Promoting sustainable consumer behavior by cueing common environmental behaviors as environmental." Interna- 
tional Journal of Research in Marketing 25 (1): 46-55.

Daniel, Florian, Pavel Kucherbaev, Cinzia Cappiello, Boualem Benatallah, and Mohammad Allahbakhsh. 2018. "Quality Control in Crowdsourcing: A Survey of Quality Attributes, Assessment Techniques, and Assurance Actions." ACM Computing Surveys 51 (1): 7:1-7:40.

Degeratu, Alexandru M., Arvind Rangaswamy, and Jianan Wu. 2000. "Consumer choice behavior in online and traditional supermarkets: The effects of brand name, price, and other search attributes." International Journal of Research in Marketing 17 (1): 55-78.

D’Souza, Clare, Mehdi Taghian, Peter Lamb, and Roman Peretiatkos. 2006. "Green products and corporate strategy: an empirical investigation." Society and business review 1 (2): 144-157.

Elahi, Mehdi, Francesco Ricci, and Neil Rubens. 2016. "A survey of active learning in collaborative filtering recommender systems." Computer Science Review 20: 29-50.

Espinosa Ceniceros, Juan Carlos, Satu Elisa Schaeffer, and Sara Elena Garza Villarreal. 2014. "Augmented reality for green consumption: using computer vision to inform the consumers at time of purchase." In Proceedings of 13th Mexican International Conference on Artificial Intelligence (MICAI 2014), Tuxtla Gutierrez, Mexico, 45-51. IEEE Computer Society, Nov..

Fang, Yu-Min, and Meng-Shien Sun. 2016. "Applying eco-visualisations of different interface formats to evoke sustainable behaviours towards household water saving." Behaviour \& Information Technology 35 (9): 748-757.

Fleith de Medeiros, Janine, José Luis Duarte Ribeiro, and Marcelo Noguiera Cortimiglia. 2016. "Influence of perceived value on purchasing decisions of green products in Brazil." Journal of Cleaner Production 110: 158-169.

Flynn, Andrew, Li Yu, Peter Feindt, and Chun Chen. 2016. "Eco-cities, governance and sustainable lifestyles: The case of the Sino-Singapore Tianjin Eco-City." Habitat International 53: 78-86.

Fraccascia, Luca, Ilaria Giannoccaro, and Vito Albino. 2018. "Green product development: What does the country product space imply?." Journal of Cleaner Production 170: 1076-1088.

Fram, Eugene H., and Joel Axelrod. 1990. "The Distressed Shopper." American Demographics 12: 44-45.

Galarraga Gallastegui, Ibon. 2002. "The use of eco-labels: a review of the literature." European Environment 12 (6): $316-331$.

Gan, Christopher, Han Yen Wee, Lucie Ozanne, and TzuHui Kao. 2008. "Consumers' purchasing behavior towards green products in New Zealand." Innovative Marketing 4: 93-201.

Gershoff, Andrew D., and Judy K. Frels. 2015. "What Makes It Green? The Role of Centrality of Green Attributes in Evaluations of the Greenness of Products." Journal of Marketing 79 (1): 97-110.

Gilg, Andrew, Stewart Barr, and Nicholas Ford. 2005. "Green consumption or sustainable lifestyles — Identifying the sustainable consumer." Futures 37 (6): 481-504. 
Goodchild, Michael F., and Linna Li. 2012. "Assuring the quality of volunteered geographic information." Spatial Statistics 1: 110-120.

Góssling, Stefan, Daniel Scott, C. Michael Hall, Jean-Paul Ceron, and Ghislain Dubois. 2012. "Consumer behaviour and demand response of tourists to climate change." Annals of Tourism Research 39 (1): 36-58.

Grunert, Klaus, Sophie Hieke, and Josephine Wills. 2014. "Sustainability labels on food products: Consumer motivation, understanding and use." Food Policy 44 (1): 177-189.

Häubl, Gerald, and Valerie Trifts. 2000. "Consumer Decision Making in Online Shopping Environments: The Effects of Interactive Decision Aids." Marketing Science 19 (1): 4-21.

He, Chen, Denis Parra, and Katrien Verbert. 2016. "Interactive recommender systems: A survey of the state of the art and future research challenges and opportunities." Expert Systems with Applications 56: 9-27.

Hossein Khorshidi, Gholam, Tohid Gholizadeh, and Arash Naghash. 2013. "Consumer Behavior in Relation to the decision to Buy Green Products: an Investigation on Iranian consumers." International Journal of Management Perspective 1 (3): 61-71.

Huseynov, Farid, Sema Yildiz Huseynov, and Sevgi Özkan. 2016. "The influence of knowledge-based ecommerce product recommender agents on online consumer decision-making." Information Development $32(1): 81-90$.

Hussain, Muhammad Azhar, Muhammad Fayyaz Khokhar, and Ali Asad. 2014. "Green Awareness Effects on Consumers' Purchasing Decision: A Case of Pakistan." Global Journal of Management and Business Research: E Marketing 14.

Javornik, Ana. 2016. "'It's an illusion, but it looks real!' Consumer affective, cognitive and behavioural responses to augmented reality applications." Journal of Marketing Management 32 (9-10): 987-1011.

Joski, Yatish, and Zillur Rahman. 2015. "Factors Affecting Green Purchase Behaviour and Future Research Directions." International Strategic Management Review 3 (1-2): 128-143.

Jung, Keechul, Kwang In Kim, and Anil K. Jain. 2004. "Text information extraction in images and video: a survey." Pattern Recognition 37 (5): 977-997.

Juwaheer, Thanika Devi, and Sharmila Pudaruth. 2001. "Analysing the impact of green marketing strategies on consumer purchasing patterns in Mauritius." World Journal of Entrepreneurship, Management and Sustainable Development 8 (1): 36-59.

Kahan, Dan M., Ellen Peters, Maggie Wittlin, Paul Slovic, Lisa Larrimore Ouellette amd Donald Braman, and Gregory Mandel. 2012. "The polarizing impact of science literacy and numeracy on perceived climate change risks." Nature Climate Change 2: 732-735.

Kalnikaitè, Vaiva, and Steve Whittaker. 2008. "Cueing Digital Memory: How and Why Do Digital Notes Help Us Remember?." In Proceedings of the 22nd British HCI Group Annual Conference on People and Computers: Culture, Creativity, Interaction, Vol. 1Liverpool, United Kingdom, 153-161. BCS Learning \& 
Behaviour \& Information Technology - FOR PEER REVIEW ONLY

Development Ltd.

Kalnikaitè, Vaiva, and Steve Whittaker. 2011. "A saunter down memory lane: Digital reflection on personal mementos." International Journal of Human-Computer Studies 69 (5): 298 - 310. Designing for Reflection on Personal Experience.

Kamarainen, Amy M., Shari Metcalf, Tina Grotzer, Allison Browne, Diana Mazzuca, M. Shane Tutwiler, and Chris Dede. 2013. "EcoMOBILE: Integrating augmented reality and probeware with environmental education field trips." Computers \& Education 68: 545-556.

Kern, Mary Rossick, and Ismail S. Abbass. 2008. "Personalized shopping assistant." US Patent Application 11/828,207, https://www.google.com/patents/US20080208705.

Kourouthanassis, Panos E., George M. Giaglis, and Adam P. Vrechopoulos. 2007. "Enhancing user experience through pervasive information systems: The case of pervasive retailing." International Journal of Information Management 27 (5): 319-335.

Laros, Fleur J.M., and Jan-Benedict E.M. Steenkamp. 2005. "Emotions in consumer behavior: a hierarchical approach." Journal of Business Research 58 (10): 1437-1445.

Lee, Jeonggyu, Siddharth Bhatt, and Rajneesh Suri. 2018. "When consumers penalize not so green products." Psychology \& Marketing 35: 36-46.

Lee, Ying-Lien, Fei-Hui Huang, and Sheue-Ling Hwang. 2009. "Green Advocate in E-Commerce." In HumanComputer Interaction. Interacting in Various Application Domains, 551-557. Springer.

Liangxing, Yu, and Dong Aihua. 2010. "Hybrid Product Recommender System for Apparel Retailing Customers." In Proceedings of the WASE International Conference on Information Engineering, Vol. 1Beidaihe, Hebei, China, 356-360. Aug..

Liu, Bing. 2012. Sentiment analysis and opinion mining. Morgan \& Claypool Publishers.

Longoni, Chiara, Peter M. Gollwitzer, and Gabriele Oettingen. 2014. "A green paradox: Validating green choices has ironic effects on behavior, cognition, and perception." Journal of Experimental Social Psychology 50 (1): 158-165.

Lu, Yuzhu, and Shana Smith. 2007. "Augmented Reality E-Commerce Assistant System: Trying While Shopping." In Human-Computer Interaction: Interaction Platforms and Techniques, Vol. 4551 of Lecture Notes in Computer Science643-652. Springer.

M. Kang, Ju-Young. 2014. "Augmented reality and motion capture apparel e-shopping values and usage intention." International Journal of Clothing Science and Technology 26 (6): 486-499.

Magnier, Lise, Jan Schoormans, and Ruth Mugge. 2016. "Judging a product by its cover: Packaging sustainability and perceptions of quality in food products." Food quality and preference 53: 132-142.

Martin, David. 2012. "Augmented reality system for product selection." Canadian Patent PCT/US2012/029,922, https://wWw.google.com/patents/W02012129291A1?cl=en. 
Massung, Elaine, David Coyle, Kirsten F. Cater, Marc Jay, and Chris Preist. 2013. "Using Crowdsourcing to Support Pro-environmental Community Activism." In Proceedings of the SIGCHI Conference on Human Factors in Computing Systems, Paris, France, 371-380. New York, NY, USA: ACM.

Menczer, Filippo, William Nick Street, and Narayan Vishwakarma. 2002. "IntelliShopper: A Proactive, Personal, Private Shopping Assistant." In Proceedings of the 1st international joint conference on Autonomous agents and multiagent systems: part 3, 1001-1008. ACM.

Miller, George A. 1956. "The Magical Number Seven, Plus or Minus Two: Some Limits on Our Capacity for Processing Information." The Psychological Review 101 (2): 343-352.

Moorman, Christine. 1990. "The Effects of Stimulus and Consumer Characteristics on the Utilization of Nutrition Information." Journal of Consumer Research 17 (3): 362-374.

Moradi, Parham, and Sajad Ahmadian. 2015. "A reliability-based recommendation method to improve trust-aware recommender systems." Expert Systems with Applications 42 (21): 7386-7398.

Musto, Cataldo, Pasquale Lops, Pierpaolo Basile, Marco de Gemmis, and Giovanni Semeraro. 2016. "Semantics-aware Graph-based Recommender Systems Exploiting Linked Open Data." In Proceedings of the 2016 Conference on User Modeling Adaptation and Personalization, Halifax, Nova Scotia, Canada, 229-237. New York, NY, USA: ACM.

Napolitano, Fabio, Ada Braghieri, Edi Piasentier, Saida Favotto, Simona Naspetti, and Raffaele Zanoli. 2010. "Effect of information about organic production on beef liking and consumer willingness to pay." Food Quality and Preference 21 (2): 207-212.

Napolitano, Fabio, Corrado Pacelli, Antonio Girolami, and Ada Braghieri. 2008. "Effect of information about animal welfare on consumer willingness to pay for yogurt." Journal of Dairy Science 91 (3): 910-917.

Nguyen, The Ninh, Antonio Lobo, and Steven Greenland. 2016. "Pro-environmental purchase behaviour: The role of consumers' biospheric values." Journal of Retailing and Consumer Services 33: 98-108.

Nguyen, The Ninh, Antonio Lobo, and Steven Greenland. 2017. "Energy efficient household appliances in emerging markets: the influence of consumers' values and knowledge on their attitudes and purchase behaviour." International Journal of Consumer Studies 41 (2): 167-177.

Nurmi, Petteri, Antti Salovaara, Andreas Forsblom, Fabian Bohnert, and Patrik Floréen. 2014. "PromotionRank: Ranking and Recommending Grocery Product Promotions Using Personal Shopping Lists." ACM Transactions on Interactive Intelligent Systems 4 (1): 1.

Ohbuchi, Eisaku, Hiroshi Hanaizumi, and Lim Ah Hock. 2004. "Barcode readers using the camera device in mobile phones." In 2004 International Conference on Cyberworlds, 260-265. IEEE Computer Society, Nov..

Pancer, Ethan, Lindsay McShane, and Theodore J. Noseworthy. 2017. "Isolated Environmental Cues and Product Efficacy Penalties: The Color Green and Eco-labels." Journal of Business Ethics 143: 159-177. 
Park, Do-Hyung, Jumin Lee, and Ingoo Han. 2007. "The Effect of On-Line Consumer Reviews on Consumer Purchasing Intention: The Moderating Role of Involvement." International Journal of Electronic Commerce 11 (4): 125-148.

Prakash, Gyan, and Pramod Pathak. 2017. "Intention to buy eco-friendly packaged products among young consumers of India: A study on developing nation." Journal of Cleaner Production 141: 385-393.

Ricci, Francesco, Lior Rokach, and Bracha Shapira. 2011. Introduction to recommender systems handbook. Springer.

Roberts, James A., and Donald R. Bacon. 1997. "Exploring the Subtle Relationships between Environmental Concern and Ecologically Conscious Consumer Behavior." Journal of Business Research 40 (1): 79-89.

Schnettler, Berta, Ricardo Vidal, Roberto Silva, Lisete Vallejos, and Néstor Sepúlveda. 2009. "Consumer willingness to pay for beef meat in a developing country: The effect of information regarding country of origin, price and animal handling prior to slaughter." Food Quality and Preference 20 (2): 156-165.

Scholz, Joachim, and Andrew N. Smith. 2016. "Augmented reality: Designing immersive experiences that maximize consumer engagement." Business Horizons 59 (2): 149-161.

Sklar, Elizabeth I., Simon Parsons, Zimi Li, Jordan Salvit, Senni Perumal, Holly Wall, and Jennifer Mangels. 2016. "Evaluation of a trust-modulated argumentation-based interactive decision-making tool." Autonomous Agents and Multi-Agent Systems 30 (1): 136-173.

Tarng, Wernhuar, and Kuo-Liang Ou. 2012. "A Study of Campus Butterfly Ecology Learning System Based on Augmented Reality and Mobile Learning." In Proceedings of the 7th International Conference on Wireless, Mobile and Ubiquitous Technology in Education, Takamatsu, Japan, 62-66. IEEE, Mar..

Tellis, Gerard J., and Gary J. Gaeth. 1990. "Best Value, Price-Seeking, And Price Aversion: The Impact of Information and Learning on Consumer Choices." Journal of Marketing 54 (2): 34-45.

Thøgersen, John, Pernille Haugaard, and Anja Olesen. 2010. "Consumer responses to ecolabels." European Journal of Marketing 44 (11/12): 1787-1810.

TNS Political \& Social. 2013. Attitudes of Europeans towards building the single market for green products. Technical Report Flash Eurobarometer 367. Directorate-General for Communication, European Commission.

Tuytelaars, Tinne, and Krystian Mikolajczyk. 2008. "Local invariant feature detectors: a survey." Foundations and Trends in Computer Graphics and Vision 3 (3): 177-280.

Urbina Coronado, Sandra Cecilia, Satu Elisa Schaeffer, and Sara Elena Garza Villarreal. 2014. "A visual aid on the ecological impact of products for online retail." Research in Computing Science 81: 103-112. Advances in Computing Science.

von Reischach, Felix, Florian Michahelles, Dominique Guinard, Robert Adelmann, Elgar Fleisch, and Albrecht Schmidt. 2009. "An Evaluation of Product Identification Techniques for Mobile Phones." In 
Proceedings of the 12th IFIP TC 13 International Conference on Human-Computer Interaction: Part I, Vol. 5726 of Lecture Notes in Computer Science804-816. Springer-Verlag GmbH.

Wang, Wen-Lan. 2012a. "Most Feasible Strategies for Green Marketing Mix under Business Sustainable Development." The Business Review 20 (1): 297-303.

Wang, Wen-Lan. 2012b. "A Study on Consumer Behavior for Green Products from a Lifestyle Perspective." The Journal of American Academy of Business 18 (1): 164-170.

Yadav, Rambalak, and Govind Swaroop Pathak. 2016. "Young consumers' intention towards buying green products in a developing nation: Extending the theory of planned behavior." Journal of Cleaner Production 135: $732-739$. 
Principal components of the proposed ecological product- recommendation framework. 
The phases of interaction between the recommendation engine and a scoring module of the architecture presented in Figure 1.

\section{$321 \times 212 \mathrm{~mm}(72 \times 72 \mathrm{DPI})$}

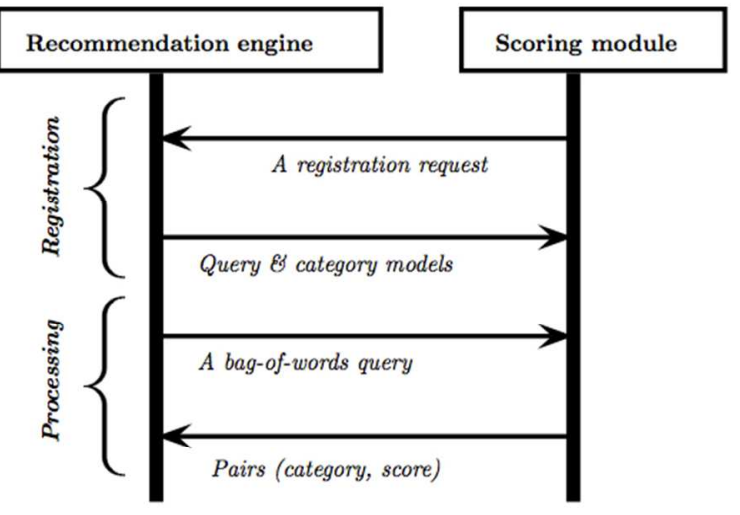




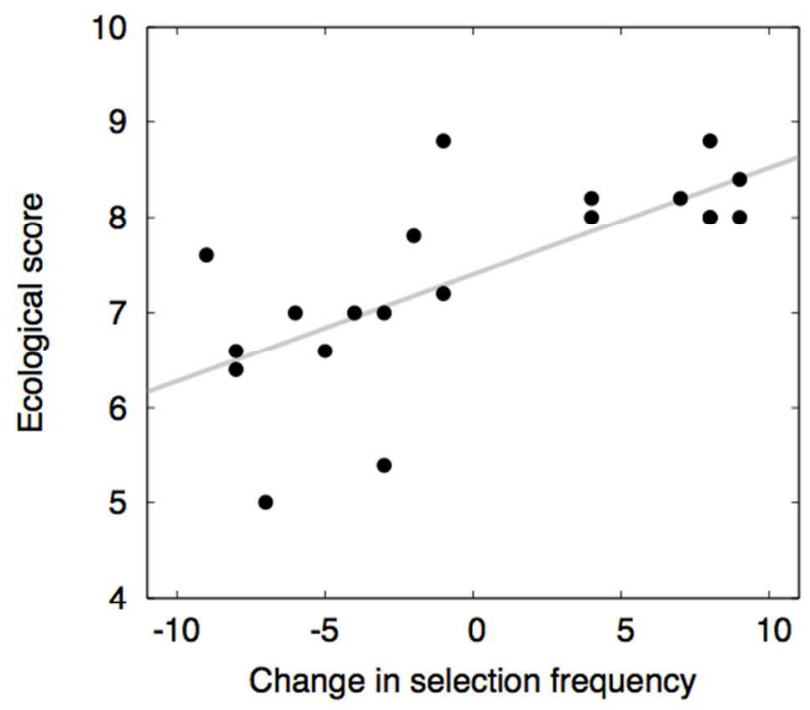

The difference in the frequency of selection of brands into a set of ten favorites, before and after using the AR prototype (the frequency of selections after the usage minus the frequency of selections of the brand before the usage - positive values indicate a change in favor of the brand whereas negative ones indicate a change against it) versus the ecological score assigned to each brand.

$301 \times 233 \mathrm{~mm}(72 \times 72 \mathrm{DPI})$ 